\title{
RF Photoinjector Development for a Short- Pulse, Hard X-Ray Thomson Scattering Source
}

G. P. Le Sage, S. G. Anderson, T. E. Cowan, J. K. Crane, T. Ditmire, J. B. Rosenzweig

This article was submitted to Advanced Accelerator Concepts 2000, Santa Fe, NM, June 10-16, 2000

Lawrence Livermore National Laboratory

August 15, 2000 


\section{DISCLAIMER}

This document was prepared as an account of work sponsored by an agency of the United States Government. Neither the United States Government nor the University of California nor any of their employees, makes any warranty, express or implied, or assumes any legal liability or responsibility for the accuracy, completeness, or usefulness of any information, apparatus, product, or process disclosed, or represents that its use would not infringe privately owned rights. Reference herein to any specific commercial product, process, or service by trade name, trademark, manufacturer, or otherwise, does not necessarily constitute or imply its endorsement, recommendation, or favoring by the United States Government or the University of California. The views and opinions of authors expressed herein do not necessarily state or reflect those of the United States Government or the University of California, and shall not be used for advertising or product endorsement purposes.

This is a preprint of a paper intended for publication in a journal or proceedings. Since changes may be made before publication, this preprint is made available with the understanding that it will not be cited or reproduced without the permission of the author.

This work was performed under the auspices of the United States Department of Energy by the University of California, Lawrence Livermore National Laboratory under contract No. W-7405-Eng-48.

This report has been reproduced directly from the best available copy.

Available electronically at http://www.doc.gov/bridge

Available for a processing fee to U.S. Department of Energy

And its contractors in paper from

U.S. Department of Energy

Office of Scientific and Technical Information

P.O. Box 62

Oak Ridge, TN 37831-0062

Telephone: (865) 576-8401

Facsimile: (865) 576-5728

E-mail: reports@adonis.osti.gov

Available for the sale to the public from

U.S. Department of Commerce

National Technical Information Service

5285 Port Royal Road

Springfield, VA 22161

Telephone: (800) 553-6847

Facsimile: (703) 605-6900

E-mail: orders@ntis.fedworld.gov

Online ordering: http://www.ntis.gov/ordering.htm

OR

Lawrence Livermore National Laboratory

Technical Information Department's Digital Library

http://www.llnl.gov/tid/Library.html 


\title{
RF Photoinjector Development for a Short- Pulse, Hard X-Ray Thomson Scattering Source
}

\author{
G.P. Le Sage ${ }^{1}$, S.G. Anderson ${ }^{2}$, T.E. Cowan ${ }^{1}$, J.K. Crane ${ }^{1}$, T. Ditmire ${ }^{1}$ and \\ J.B. Rosenzweig ${ }^{2}$
}

1. University of California, Lawrence Livermore National Laboratory, Livermore CA 94550 USA

2. UCLA Department of Physics and Astronomy, Los Angeles CA 90059 USA

\begin{abstract}
An important motivation in the development of thenext generation $x$-ray light sources is to achicve picosecond and sub-ps pulses of hard x-rays for dynamic studies of a varicty of physical, chemical and biological processes. Present hard x-ray sources are either pulse-width or intensity limited, which allows ps-scale temporal resolution only for signal averaging of highly repetitive processes. A much faster and brighter hard $x$-ray source is being developed at LLNL, based on Thomson scattering of fs-laser pulses by a relativistic electron beam, which will enable $\mathrm{x}$-ray characterization of the transient structure of a sample in a single shot. Experimental and diagnostic techniques relevant to the development of next generation sources including the Linac Coherent Light Source can be tested with the Thomson scattering hard x-ray source. This source will combine an RF photoinjector with a $100 \mathrm{MeV}$ S-band linac. The photoinjector and linac also provide an ideal test-bed for examining space-charge induced emittance growth effects. A program of beam dynamics and diagnostic experiments are planned in parallel with Thomson source development. Our experimental progress and future plans will be discussed.
\end{abstract}

\section{INTRODUCTION}

The use of ultrafast laser pulses to generate high brightness, ultrashort pulses of $x$ rays is a topic of interest to the $\mathrm{x}$-ray user community. Femtosecond-scale pumpprobe experiments can in principle be used to temporally resolve structural dynamics of materials on the time scale of atomic motion. A Thomson scattering source is being developed at LLNL to provide ultrashort ( $\mathrm{ps}$ to fs) $\mathrm{x}$-rays. With this machine we intend to improve on the performance of a previously demonstrated Thomson source at LBNL in which $300 \mathrm{fs}, 30 \mathrm{keV}$ pulses were generated $[1,2]$. Our ultimate goal is to increase the $\mathrm{x}$-ray brightness by four to five orders of magnitude.

Using a short-pulse laser, RF linac, and photoinjector, the LLNL source will provide a means of performing pump-probe experiments on a sub-picosecond time scale, with flux suitable for single-shot measurements. Single-shot measurement enables experiments on samples undergoing irreversible damage: shocks, plasma ablation, or ultrafast melting.

The LLNL Thomson scattering x-ray source combines a 35 fs laser system, currently producing $0.6 \mathrm{~J}$ at a $1 \mathrm{~Hz}$ repetition rate, with a planned upgrade to $4 \mathrm{~J}$ with a $0.1 \mathrm{~Hz}$ repetition rate, a photoinjector capable of producing $5 \mathrm{MeV}$ electron pulses with charge up to $1 \mathrm{nC}$, pulse length of $0.2-10 \mathrm{ps}$, and normalized, rms emittance of 5 
$\pi$ mm-mrad, and an RF linac with output energy adjustable from 30 to $100 \mathrm{MeV}$. Each subsystem is described below, including current progress and future plans.

\section{PHOTOINJECTOR}

The LLNL Photoinjector uses the BNL/SLAC/UCLA 1.6 cell, standing wave accelerator geometry [3] The photoinjector cavity and diamond-turned cathode were constructed using High Isostatic Pressure annealed OFHC Copper [4]. The photocathode surface has a measured flatness of $79 \mathrm{~nm}$ peak-to-valley over a 2 inch diameter $(\lambda / 8$ at $633 \mathrm{~nm})$. With the experience of the groups involved in the construction of previous photoinjectors, development of the LLNL photoinjector progressed quickly. First metal cutting for the photoinjector began in December of 1998 at the UCLA Physics department precision machine shop. High power RF was applied to the photoinjector in July of 1999. The first photoelectron beam was produced in January of 2000 .

In order to test the field profile of the photoinjector with the actual photocathode in place, a "bead-drop" was performed where a dielectric bead was hung from the end of a thin nylon line and lowered into the central axis of the photoinjector cavity. The usual technique requires a second test cathode plane to be made with a hole in the center for a string to pass through and suspend a bead in the horizontal direction: "bead-pull." The result of the bead-drop measurement is presented in Fig. 1, in comparison to the field profile generated by SUPERFISH, with a background image of the $\mathrm{\rho H}_{\mathrm{o}}$ profile.

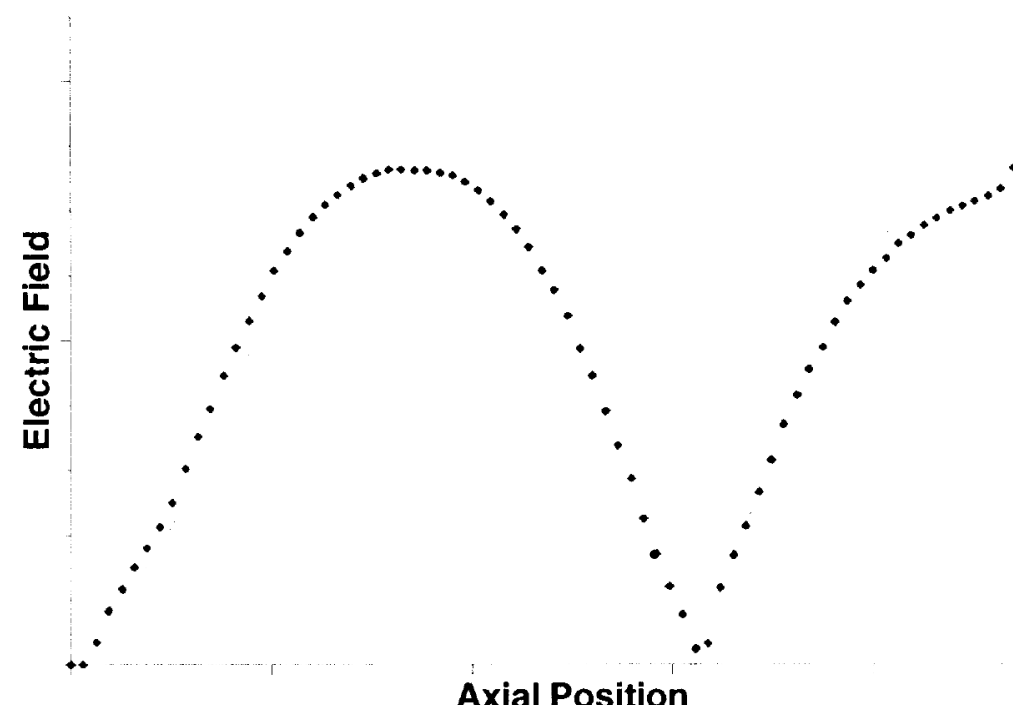

Gun exit

Cathode

FIGURE 1. Bead-drop characterization of photoinjector ficld profile.

The photoinjector is currently operated separately from the RF linac, and has been fully characterized using a diagnostic beamline. Characterization of the photoinjector 
has included measurement of cavity $Q$ and filling time, dark current, charge and quantum efficiency, Schottky scan, energy, and emittance. Emittance has also been characterized as a function of charge and pulse length.

The energy range of field emitted electrons (dark current) in the cavity ranges broadly up to the peak beam energy. Characterization of the total field emission through current collection with a Faraday cup or integrating current transformer is subject to beam dynamics issues as the RF power and beam energy changes. An equivalent measurement of the Fowler-Nordheim macroscopic field enhancement factor $\beta$ can be made by measuring the radiation produced by the gun as a function of $\mathrm{RF}$ drive power [5]. A time-averaging radiation meter was placed at approximately half a meter from the photoinjector and measured radiation levels up to $500 \mathrm{mR} / \mathrm{h}$ with $\mathrm{RF}$ drive power up to $7 \mathrm{MW}$. An image of the dark current emission, focused by the photoinjector solenoids, and captured on a scintillator shows the emission from the cathode surface and the iris between the two gun cells in Fig. 2.

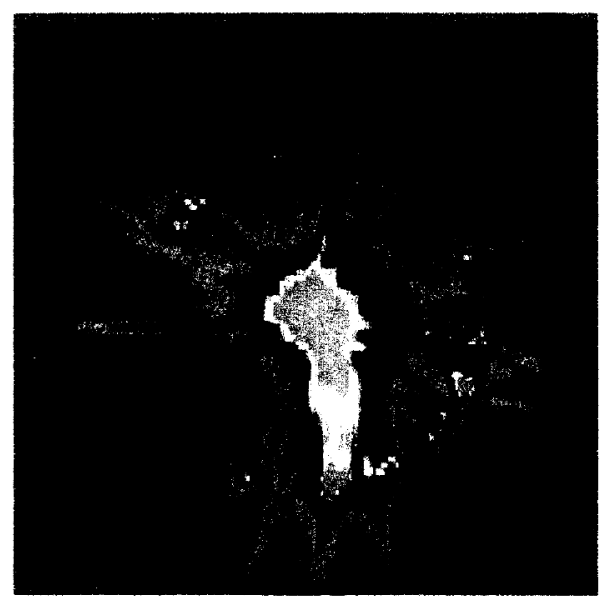

FIGURE 2. Dark current imaged with photoinjector solenoids.

A plot of 1/E versus the logarithm of the dose rate divided by $E^{4}$ is shown in Fig. 3, and provides the means of measuring the field enhancement factor $\beta$. The $\beta$ value measured for the LLNL HIP Copper photoinjector, taken from the slope of this plot was 62. 


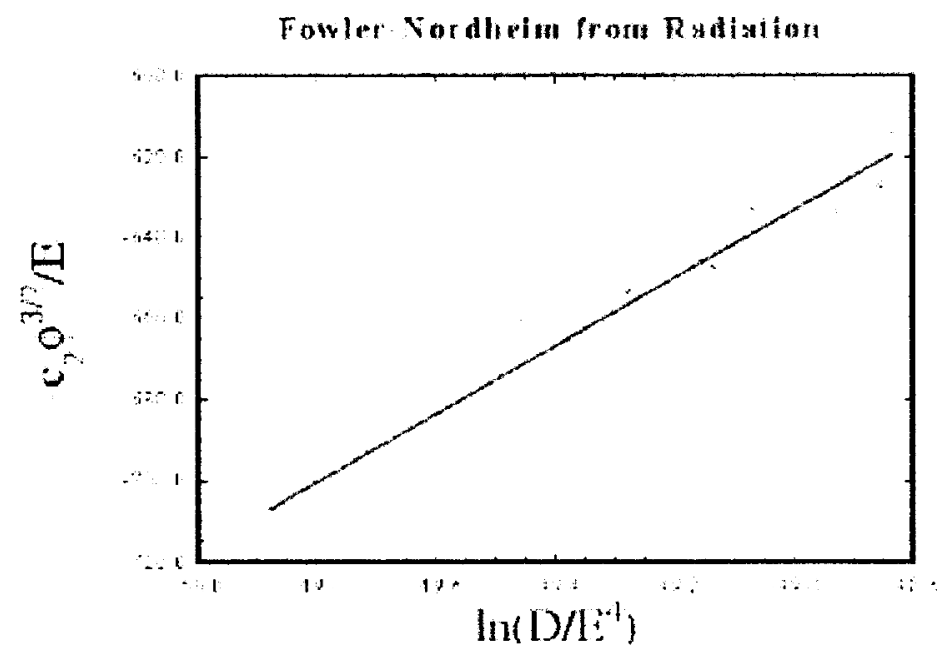

FIGURE 3. Fowler-Nordheim equivalent field emission plot.

The photoinjector cavity Ohmic $Q$ was measured in cold test before and after brazing, and had a final cold-test value of 13,000. The value predicted by SUPERFISH, taking into account the loss of the Copper surface including the cathode plane was 15,878 . The $Q$ value of 13,000 was also verified by examining the filling time of the RF cavity with high power RF applied. The cavity fills with the characteristic $Q_{0}$ and empties with $Q=\left[1 / Q_{0}+1 / Q_{e}\right]^{-1}=\frac{1}{2} Q_{0}$ as shown in Fig. 4.

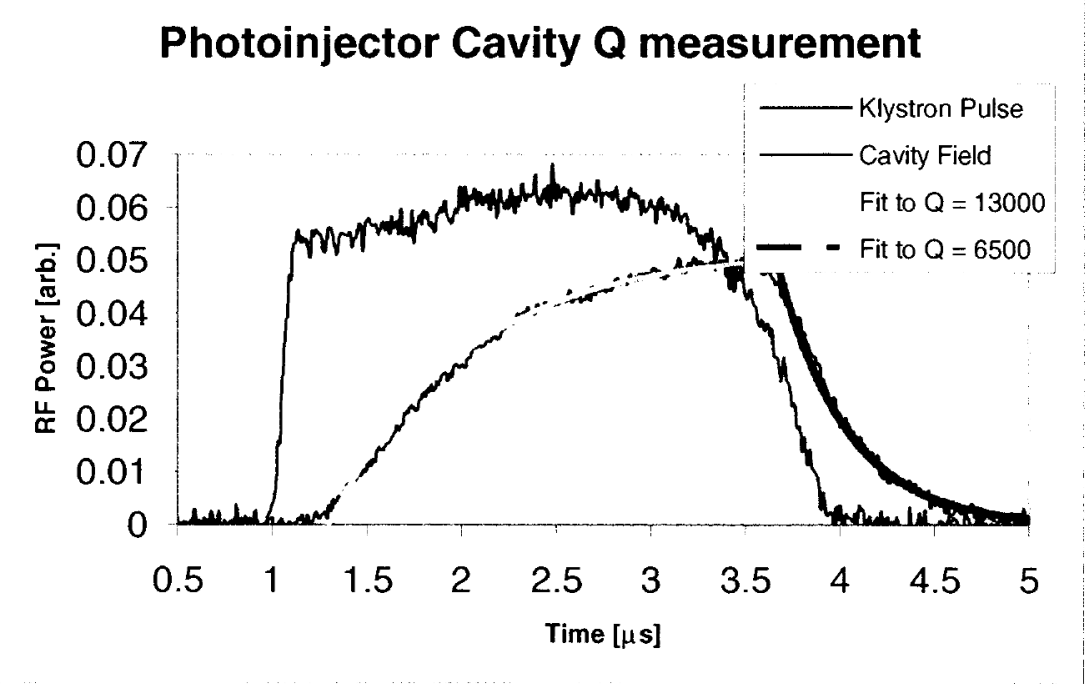

FIGURE 4. High power measurement of photoinjector cavity $Q$.

A summary of the LLNL photoinjector RF characteristics is presented in Table 1. The drive power and cathode peak field gradient correspond to production of a $5 \mathrm{MeV}$ photoelectron beam. 


\begin{tabular}{lc}
\hline \multicolumn{2}{l}{ Table 1. Photoinjector cavity parameters. } \\
\hline Beam parameter & Measured value \\
\hline Pcak Cathode Gradient & $112 \mathrm{MV} / \mathrm{m}$ \\
Drive Power & $7.1 \mathrm{MW}$ \\
Ohmic Q & 13,000 \\
Cavity $\left|\mathrm{S}_{11}\right|$ & Iess than $-30 \mathrm{~dB}$ \\
Fowler Nordheim $\beta$ parameter & 62 \\
\hline
\end{tabular}

Photoelectron current was first measured on January 21, 2000. The program of beam measurements that followed this first beam production included Schottky scanning, in order to determine the injection phase for optimum emittance, and measurement of the total charge and quantum efficiency. An image of one of the first photoelectron beam profiles measured with the system is shown in Fig. 5.

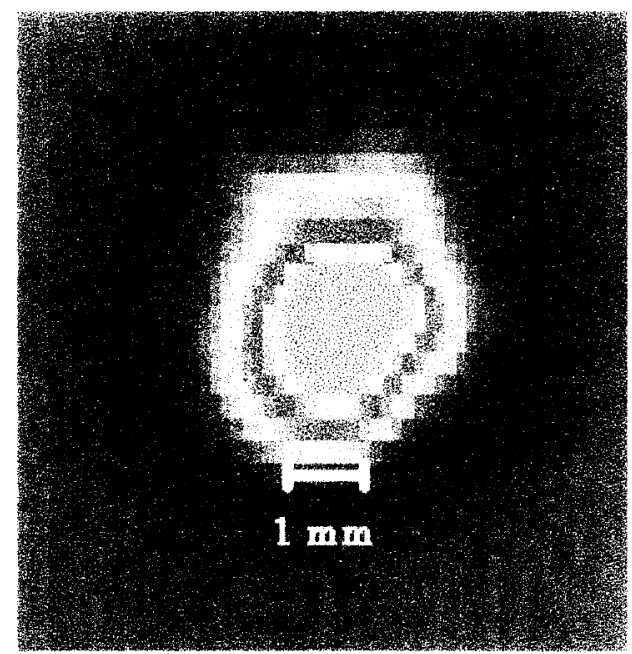

FIGURE 5. Photoelectron beam profile at $1 \mathrm{~m}$ from cathode.

The photoelectron bunch charge has been measured using both Faraday cups and a fast integrating current transformer. The peak charge per pulse that has been measured to date is $0.4 \mathrm{nC}$, and was purposely limited due to the small UV spot size on the photocathode. A full $\mathrm{nC}$ of charge extraction at high gradient with a small spot size would approach the region where cathode damage can result [6].

The quantum efficiency of the photoinjector operating at 23 phase angle off of the RF crest was measured as $2.210^{-5}$ using an integrating current transformer positioned directly at the photoinjector output, and a calibrated UV energy meter monitoring a split beam from the photocathode laser path. The required UV energy for $1 \mathrm{nC}$ of charge is thus $300 \mu \mathrm{J}$. The UV laser system has produced up to $400 \mu \mathrm{J}$ in its present configuration. The IR ( $805 \mathrm{~nm}$ wavelength) pulse length produced by the laser

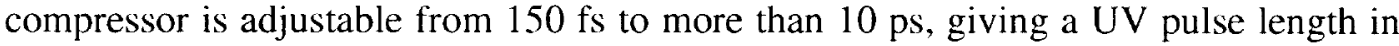
the range from less than $100 \mathrm{fs}$ to more than $9 \mathrm{ps}$. The electron pulse length has not been verified with a streak camera to date, and the electron pulse length is currently assumed to equal the UV pulse length.

The electron beam energy was measured using the steering magnets that are part of the diagnostic beamline. The steering magnet profile was integrated over a long 
longitudinal range extending into the fringe field region to give more accurate prediction of steering versus beam energy and magnet current. A linear fit of several magnet deflections, with variation of the RF drive power confirm a beam energy of $4.95 \mathrm{MeV}$ at the current RF drive power level of 7.1 MW.

The photoinjector electron beam emittance has been measured using a slit collimator [7]. This technique removes space charge from the beam measurement, and provides a single-shot means of measuring emittance through computer control and analysis. Launch phase and solenoid settings were optimized in real time using this diagnostic. The emittance as a function of beam launch parameters was characterized over a wide range of charge, pulse length, and beam radius on the photocathode. A schematic of the slit collimator arrangement is shown in Fig. 6. The collimator-slit widths are $50 \mu \mathrm{m}$, with collimator thickness is $5 \mathrm{~mm}$, and the drift length is $53 \mathrm{~cm}$.

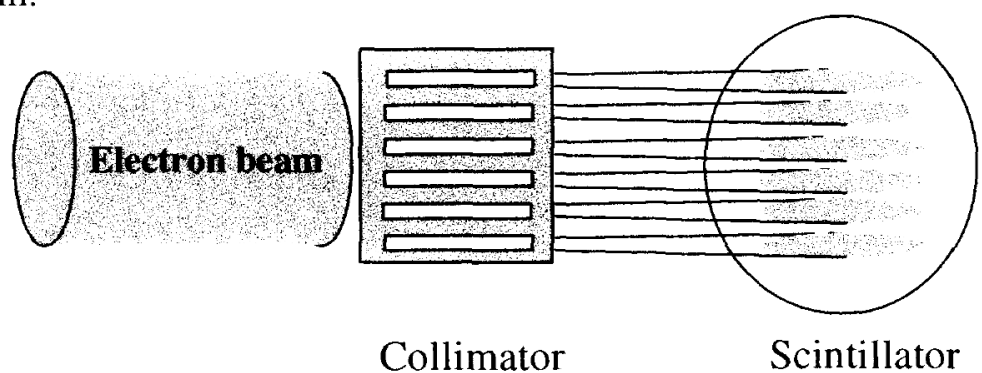

FIGURE 6. Collimator slit projection schematic.

An image of the photoelectron beam collected by the scintillator is shown in Fig. 7, and the virtual instrument panel for the emittance diagnostic is shown in Fig. 8.

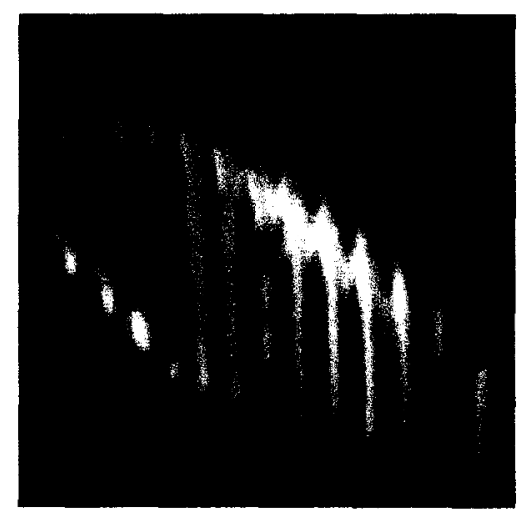

FIGURE 7. Collimator slit projection of photoinjector beam. 


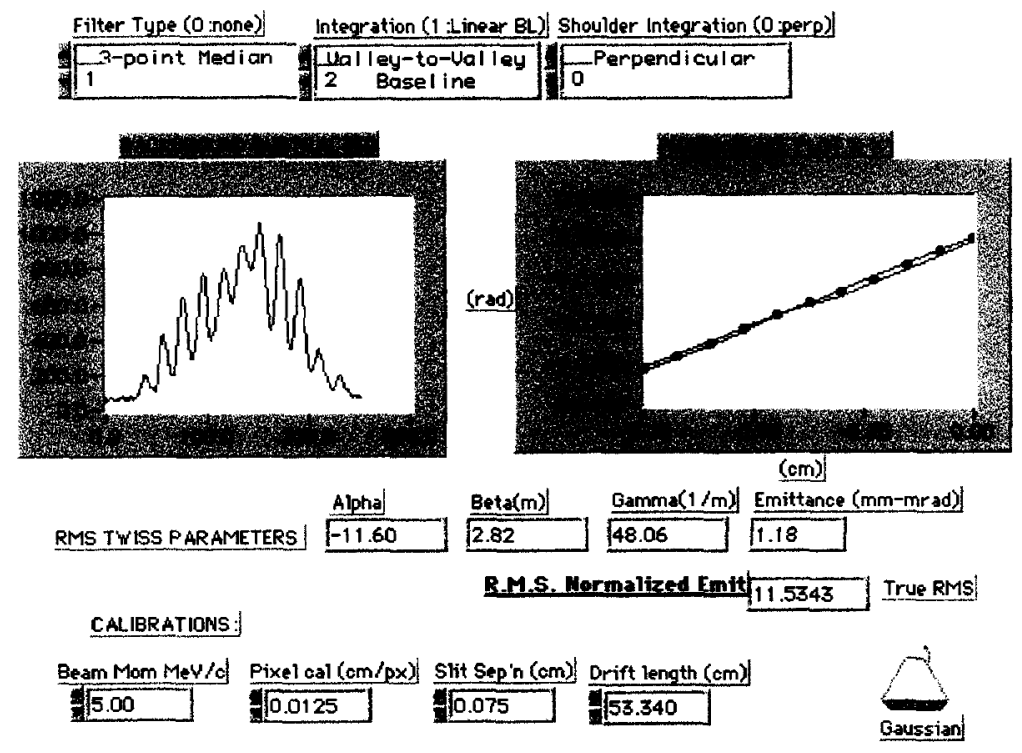

FIGURE 8. Slit emittance measurement virtual instrument panel.

The characteristics of the photoinjector for a specific pulse length are listed in Table 2. The measured emittance should be sufficient for the planned Thomson scattering source at $100 \mathrm{MeV}$, provided that excessive emittance growth through the linac is not encountered. The design value for the normalized emittance is $10 \pi \mathrm{mm}$ mrad, which implies a physical emittance at $100 \mathrm{MeV}$ of $\sim 51 \mu \mathrm{m}$-mrad. With a $20 \mathrm{~cm}$ focal length final electron optic, and a $1 \mathrm{~mm}$ entrance spot radius ( $5 \mathrm{mr}$ convergence) the emittance limited spot size is $\sim 10 \mu \mathrm{m}$. Since the focal spot size of the FALCON laser beam with an F/10 optic is estimated to be $20 \mu \mathrm{m}$, the electron focal spot should overlap well with the laser spot.

\begin{tabular}{|c|c|}
\hline Beam parameter & Measured value \\
\hline Beam energy & $5 \mathrm{MeV}$ \\
\hline UV laser pulse length & $7.1 \mathrm{ps}$ FWHM \\
\hline Bunch charge & $200 \mathrm{pC}$ \\
\hline $\begin{array}{l}\text { Emittance }_{x, \text { rns, }}, \\
\text { Quantum efficiency }\end{array}$ & $\begin{array}{c}5.8 \pi \mathrm{mm} \text {-mrad } \\
2.2 \times 10^{-5} \text { at } 23^{\circ} \text { off } \mathrm{RF} \text { crest }\end{array}$ \\
\hline
\end{tabular}

\section{LASER SYSTEM}

There are two laser systems included in the Thomson scattering project. The first is the high power, short pulse "FALCON" laser [8,9] which produces 35 fsec, $0.6 \mathrm{~J}$ pulses at $1 \mathrm{~Hz}$ repetition rate. This laser system is based on Ti:Sapphire, and relies on chirped pulse amplification. The ultimate specification goal for the FALCON laser is $4 \mathrm{~J}$ pulse energy $(\sim 100 \mathrm{TW})$ with a repetition rate of $0.1 \mathrm{~Hz}$ using two $15 \mathrm{~J}$ custombuilt Nd:glass pump lasers. In addition to the Thomson scattering project, the FALCON laser is used for a variety of laser-plasma and solid target interaction experiments. A second laser system takes seed pulses from the FALCON master 
oscillator and amplifies them for conversion to UV and photoelectron production. When the FALCON laser, photoinjector, and linac are integrated, the same laser pulse from the oscillator that is amplified through the FALCON laser amplifier chain will also be amplified by the photoinjector laser system to produce electron pulses.

The FALCON oscillator, which drives both the high power laser amplifiers and the photoinjector UV laser system is a Kerr-lens modelocked, Ti:Sapphire system with a feedback loop controlling the cavity length and thus pulse repetition frequency. The feedback system controls $\mathrm{kHz}$ level thermal and mechanical drifts. The laser oscillator is not actively modelocked by an RF master oscillator, but instead provides the master RF signal to the entire linac and photoinjector system.

\section{MeV RF LINAC}

The LLNL RF linac in its present configuration is composed of five travelling wave accelerator sections, a thermionic injector and a travelling wave buncher. The travelling wave accelerator sections are iris-loaded waveguides, and each section has an overall length of $2.6 \mathrm{~m}$, boosting the beam energy by up to $30 \mathrm{MeV}$. The thermionic injector is a pulsed DC thermionic source, followed by a travelling wave buncher that accelerates the beam from $150 \mathrm{keV}$ to $3 \mathrm{MeV}$ in a total length of $0.75 \mathrm{~m}$. The original purpose of the linac was to produce high average currents of electrons, positrons, and intense pulse neutrons. The machine is thus capable of very high repetition rates with long macropulses. In "long pulse mode" (up to $2.8 \mu \mathrm{s}$ macropulses), the linac produces roughly 8000 micropulses per RF macropulse, with a total of $2 \mu \mathrm{C}$ per pulse, at a repetition rate up to $300 \mathrm{~Hz}$. In this configuration, the linac can produce $0.6 \mathrm{~mA}$ of average current, and $0.7 \mathrm{~A}$ during each RF macropulse. In "short pulse mode" (2-20 ns macropulses), the linac can operate at a repetition rate of $1440 \mathrm{~Hz}$, producing a macropulse current of $10 \mathrm{~A}$, and an average current of 173 $\mu \mathrm{A}$. The linac beam energy has been tuned over the range of 13 to $130 \mathrm{MeV}$, and the emittance at $100 \mathrm{MeV}$ has been measured as $260 \pi \mathrm{mm}$ - $\mathrm{mrad}_{\mathrm{x}, \mathrm{rms}, \mathrm{n}}$ integrated over one macropulse, using the thermionic injector. While the high average current is important for the continuing production of positron beams at the LLNL facility, the Thomson scattering experiment relies on the low beam emittance provided by the photoinjector, and requires a repetition rate of $10 \mathrm{~Hz}$ or less, matched to the laser system.

A magnetic beam switchyard has been designed to pass the thermionic injector beam around the photoinjector when high average current operation is desired. In this way, the capabilities of both injector systems will be maintained by the LLNL accelerator facility.

\section{SYNCHRONIZATION}

Thomson scattering the FALCON laser pulse with the linac electron beam requires synchronization on the picosecond to sub-picosecond time scale. To date, synchronization between an RF linac and modelocked laser system has been 
demonstrated with an rms jitter of 2.2 ps by the E-144 collaboration at SLAC [10]. In this case, linac RF was used to actively modelock a laser oscillator, which in turn produced laser pulses for the Thomson scattering interaction. The LLNL system instead uses a laser oscillator that also functions as the master RF oscillator at the $35^{\text {th }}$ subharmonic of the linac RF. The ultimate timing jitter between the laser pulses and electron pulses will be due to long time scale contributions: thermal and mechanical in transport, and to fast time scale sources: jitter inherent to laser master oscillator, and phase noise introduced in the RF system. Assuming that the slow time scale contributions can be remedied by feedback loops with $\mathrm{kHz}$ bandwidth, the ultimate limiting factor for final synchronization rests with inherent laser oscillator jitter and RF phase noise. The LLNL system can improve upon previous synchronization results for three key reasons described as follows.

First, the laser-electron jitter that arises from the inherent jitter of the laser master oscillator will be minimized. Using the laser master oscillator as the source of the linac RF allows the linac RF system to track laser oscillator phase changes up to the characteristic frequency of the entire system. The question then becomes how fast the RF system can respond to changes in the laser-produced RF phase. The photoinjector cavity bandwidth is the limiting factor since its Ohmic Q is 13,000 (filling time is 725 $\mathrm{ns})$. The response time is thus on the order of $1 \mu \mathrm{s}$, compared to RF amplifier bandwidths, travelling wave accelerator filling times, and transmission line path lengths, each of which add up to 10 's to 100 's of ns. The phase noise integration bandwidth for the LLNL system corresponds to at most microseconds of reaction time.

The timing jitter of the laser pulses produced by a laser oscillator can be characterized by integrating laser RF harmonic spectral noise [11]. A high-bandwidth photodiode and a spectrum analyzer were used to make the jitter characterization of the FALCON laser oscillator. The integration bandwidth is determined in the case of an actively (externally) modelocked laser slaved to an RF system by the time between application of the modelocking RF signal and the final interaction of the laser and electron pulses. The integration bandwidth is larger, so the jitter is proportionately larger. Additionally, if a master RF oscillator is used to both modelock a laser and drive the linac RF system, jitter inherent to the laser oscillator driven by a perfect RF sine wave has no chance of being tracked by the RF system over any bandwidth.

The LLNL (FALCON) laser oscillator was characterized with an inherent jitter of < $1.5 \mathrm{ps}$ integrating over $1 \mu \mathrm{s}$ to $1 \mathrm{~ms}$, and a jitter of $5.5 \mathrm{ps}$ integrating over $>1 \mathrm{~ms}$. The noise floor of the detector limited the accuracy of this measurement, and $1.5 \mathrm{ps}$ jitter should be considered a worst case estimate of ultimate system performance. The spectrum of the $22^{\text {nd }}$ harmonic of the FALCON oscillator $81 \mathrm{MHz}$ RF is shown in Fig. 9. The noise floor was $-70 \mathrm{~dB}$ at frequencies more than $1 \mathrm{kHz}$ from the harmonic peak, and the resolution bandwidth was $100 \mathrm{~Hz}$. 


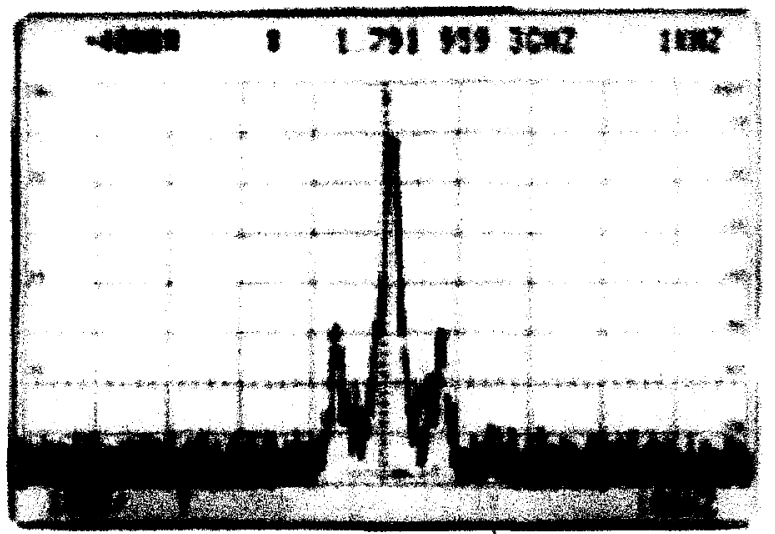

FIGURE 9. Laser oscillator spectrum of $22^{\text {nd }}$ harmonic of $81 \mathrm{MHz}$ RF.

At the SLAC Gun Test Facility, jitter measurements were made using an independent RF source to drive both a modelocked laser and the RF system, and using the laser as the master reference [12]. The laser-electron jitter was measurably lower in the case where the laser provided the master RF signal than in the case where the laser and RF systems were driven by an external reference, demonstrating the principle described here.

The second reason why the LLNL system will achieve low jitter is that phase and amplitude control of each linac section can be accomplished at the $\mathrm{mW}$ RF power level, allowing fast electronic feedback control of amplitude and phase. Each linac section and the photoinjector are driven separately by a total of six Klystron amplifiers, each in turn is driven by a booster amplifier with $\mathrm{mW}$ level RF reference input. For systems where power is split in waveguide networks between accelerator sections, mechanical phase and amplitude control is implemented, allowing only for < $\mathrm{kHz}$ level corrections at. Short feedback loops built around individual Klystrons will allow phase and amplitude flattening on a time scale short compared to the several $\mu$ slong RF macropulses.

Finally, the laser transport and RF and beam transport systems are arranged so that the same laser pulse from the FALCON master oscillator that produces the photoelectron pulse also gets amplified for the final Thomson scattering interaction. Shot-to-shot laser pulse jitter thus does not add directly to the jitter of the overall system.

\section{THOMSON SCATTERING AT $5 \mathrm{MeV}$}

The first demonstration of Thomson scattering is planned using the $5 \mathrm{MeV}$ beam directly from the photoinjector interacting with a $30 \mathrm{~mJ}$ IR laser pulse produced by the residual energy of the photocathode laser system. The interaction with the $5 \mathrm{MeV}$ beam is expected to produce $10^{4}$ to $10^{5} 600 \mathrm{eV}$ photons per shot with an interaction angle of 135 degrees between the electron and laser beams [13]. A drawing of the 5 $\mathrm{MeV}$ Thomson scattering experiment beamline is shown in Fig. 10. 


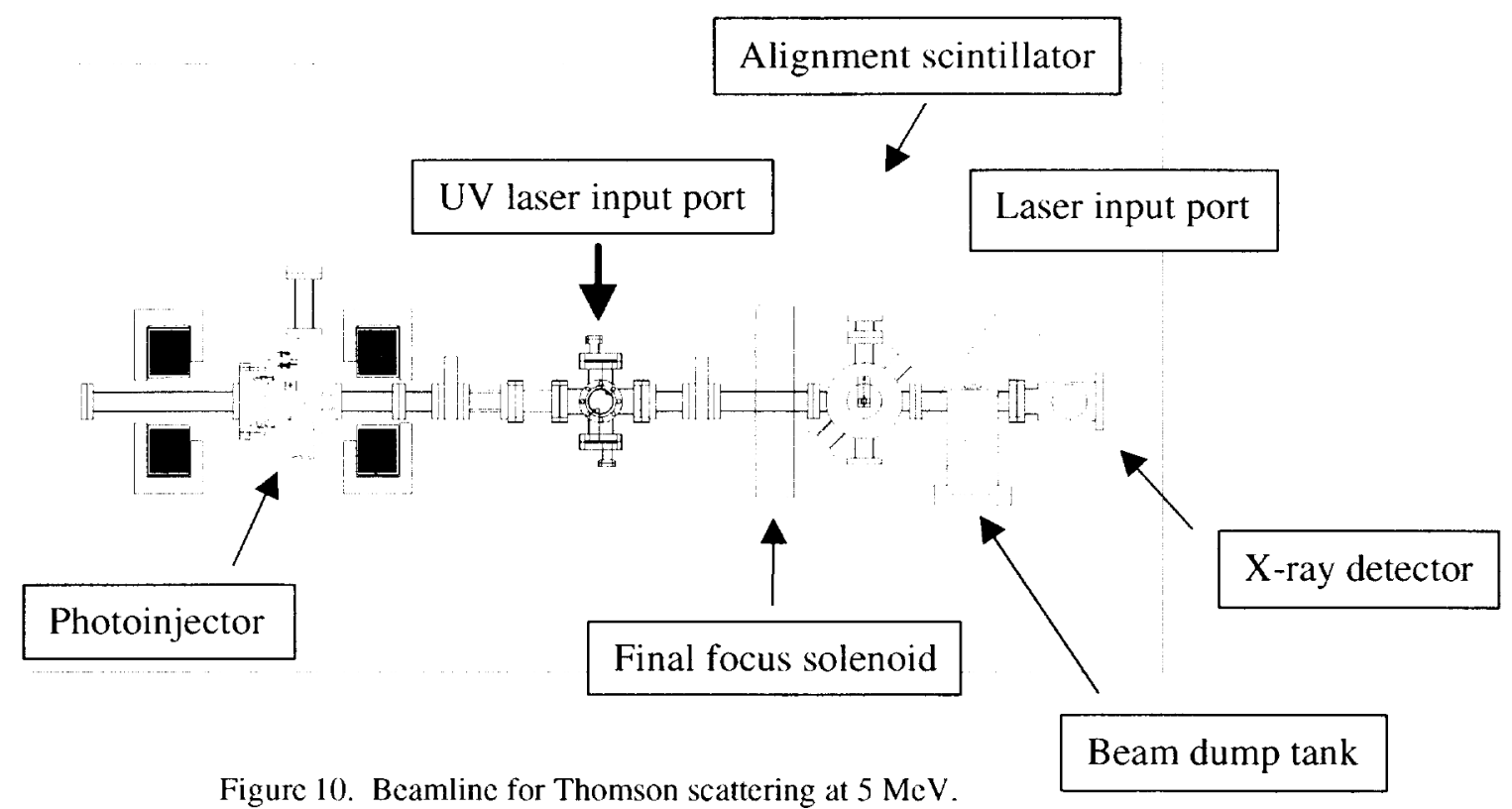

A high field solenoid was chosen for the final focusing of the $5 \mathrm{MeV}$ electron beam for the initial Thomson scattering experiment. The peak field for the final focus magnet is $3 \mathrm{kG}$, with a yoke radius of $2 \mathrm{~cm}$, and a length of $7.5 \mathrm{~cm}$. The focal length at $5 \mathrm{MeV}$ is $18 \mathrm{~cm}$. Final focus parameter optimization involves a tradeoff between the competing effects of emittance, energy spread, and spherical aberration. The scaling of these effects can be represented by a quadrature addition [14].

$$
R_{\text {final }}=\left(\frac{\varepsilon}{R_{0}} f_{0}\right)^{2}+R_{0}{ }^{2}\left(\frac{\Delta f}{f_{0}}\right)^{2}+\left(A R_{0}{ }^{3}\right)^{2}
$$

Where $R_{\text {final }}$ depends on the beam radius entering the solenoid $R_{0}$, the emittance $\varepsilon$, the lens focal length $f_{0}$, the chromatic spread $\Delta f / f_{0}$ related to the energy spread, and the spherical aberration term $A$. The spherical aberration term is determined by integrating the magnetic field through the solenoid at different radii. The POISSON simulated fields were utilized for this calculation. Using the parameters of our photoinjector and beamline, the competing effects and the total final radius were plotted as a function of the beam radius entering the final focus solenoid as shown in Fig. 11. This model assumes a continuous beam with uniform characteristics. Because of the photoinjector beam dynamics, entering the final solenoid with the optimum radius based on this model increases the energy spread significantly, which in turn changes the chromatic term. The model does however demonstrate that the optimization for the $5 \mathrm{MeV}$ experiment is achieving the minimum energy spread. Optimizing for this parameter, PARMELA simulations show that the configuration planned for the experiment should focus a $100 \mathrm{pC}, 2 \mathrm{ps}$ electron pulse to a radius of approximately $50 \mu \mathrm{m}$ ( $\sim 90 \%$ of particles). 


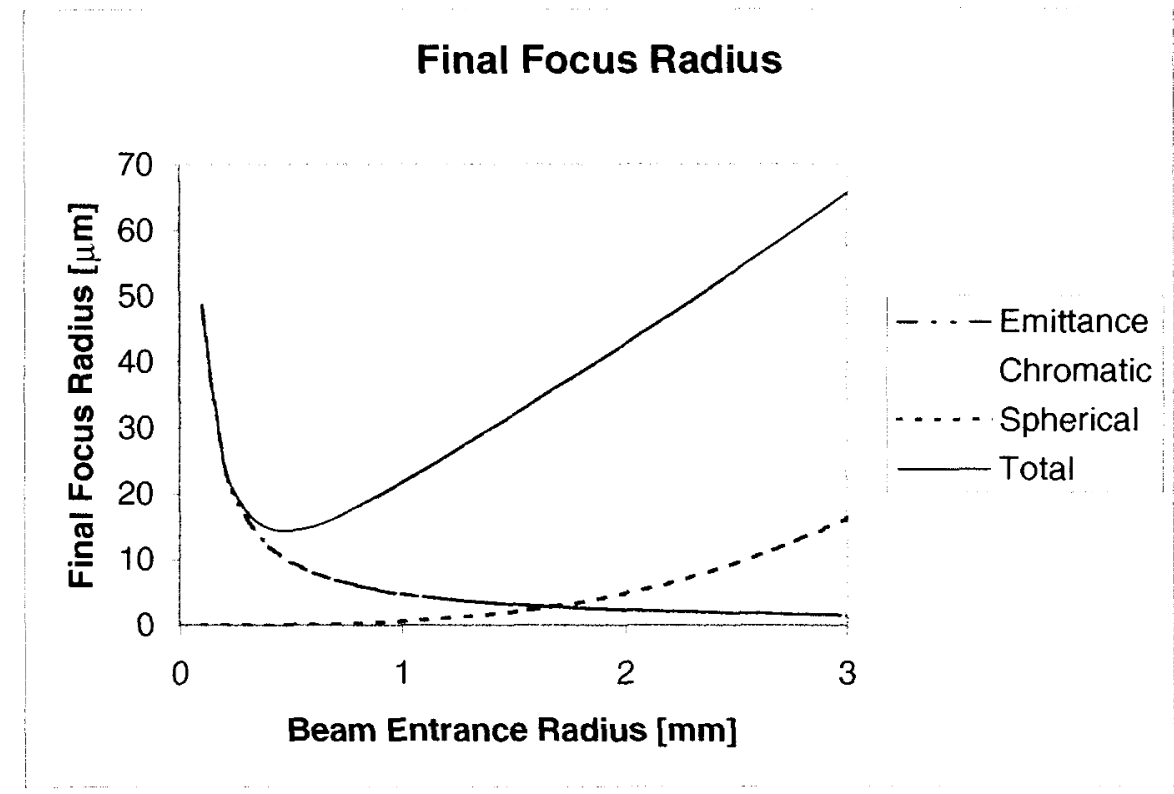

Figure 11. Final Focus calculations at $5 \mathrm{MeV}$.

With a $3 \mathrm{~mm}$ beam radius at the input of the final focus solenoid, the emittance, chromatic, and spherical aberration effects contribute $1.6 \mu \mathrm{m}, 64 \mu \mathrm{m}$, and $16 \mu \mathrm{m}$ respectively to the final spot radius. The energy spread $\Delta y$ decreases significantly with acceleration for short electron pulses. Therefore, at the future operating conditions of $100 \mathrm{MeV}$, the emittance can be expected to have a more dominant effect on the final focus spot size.

\section{BEAM DYNAMICS AND DIAGNOSTIC EXPERIMENTS}

In addition to the Thomson scattering experiment, the photoinjector and $100 \mathrm{MeV}$ RF linac also provide an ideal test-bed for examining space-charge induced emittance growth effects. A program of beam dynamics and diagnostic experiments are planned in parallel with Thomson source development for both the photoinjector, and the linac. So far a new emittance measurement technique has been demonstrated on the RF linac in order to characterize the beam produced by the thermionic injector. Based on optical transition radiation interferometry (OTRI), the emittance measurement system images and optically masks the OTRI produced by the electron beam passing through a pair of thin foils [15]. Scanning the optical mask through the electron beam allows the divergence to be measured as a function of position within the beam envelope. A proof of principle experiment was conducted to show the differences in divergence and average direction of the beam particles as a function of transverse position within the beam. Determination of the phase-space ellipse tilt using this technique changed the measured value of emittance by $45 \%$ compared to the assumption that the beam was exactly at a waist. This technique also allows real time tuning of the beam 
convergence at the diagnostic foil plane. This new diagnostic technique will be used to optimize the Thomson scattering interaction at $100 \mathrm{MeV}$.

Using the photoinjector, measurements of the emittance using the quad scan technique and slit collimator technique are being compared as a function of plasma wavelength at the photocathode. The LLNL system can be used to investigate a wide range of plasma parameters since the IR pulse length is adjustable from $150 \mathrm{fs}$ out to several ps. Since the quad-scan technique of emittance measurement is susceptible to space charge forces at low beam energy, the quad-scan and slit collimator emittance results are expected to diverge as the plasma wavelength decreases. This measurement and its implications are currently being examined.

\section{Slit Collimator Emittance Scaling}

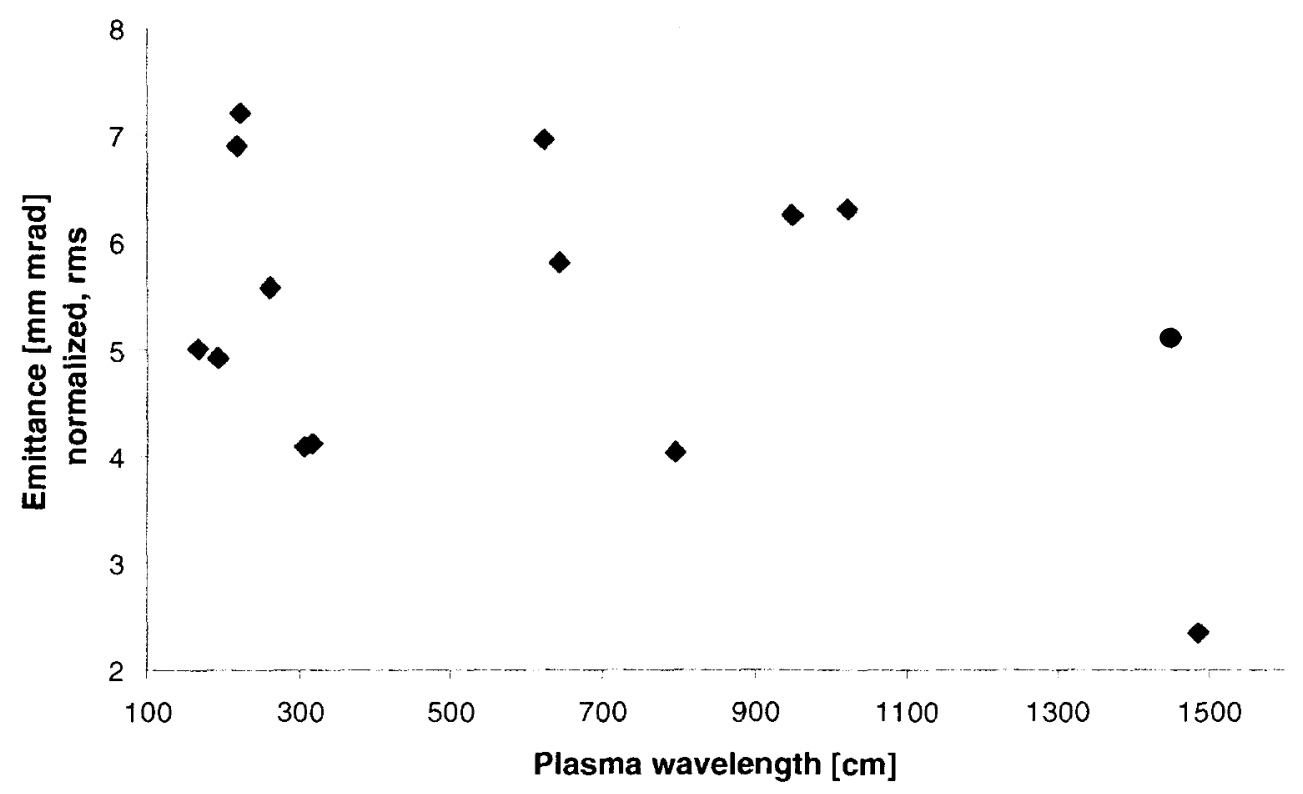

FIGURE 12. Photoinjector beam emittance scaling with plasma wavelength.

\section{SUMMARY}

Present hard x-ray sources are either pulse-width or intensity limited, which allows ps-scale temporal resolution only for signal averaging of highly repetitive processes. A Thomson scattering source is being developed at LLNL to provideultrashort (ps to fs) x-rays. Using a short-pulse laser, RF linac, and photoinjector, the source will provide a means of performing pump-probe experiments on a sub-picosecond time scale, with flux suitable for single-shot measurements. Experimental and diagnostic techniques relevant to the development of next generation sources including LCLS can be tested with the Thomson scattering hard $\mathrm{x}$-ray source. This source will combinean RF photoinjector with a $100 \mathrm{MeV}$ S- band linac and will also provide an ideal test-bed 
for examining space-charge induced emittance growth effects. A program of beam dynamics and diagnostic experiments are planned in parallel with Thomson source development.

After the Thomson scattering demonstration at $600 \mathrm{eV}$ is complete, the next major step is the installation of the photoinjector on the Linac. Propagating the electron beam through the linac, and characterization of the $100 \mathrm{MeV}$ beam will follow photoinjector installation. Compression of the FALCON beam, and delivery of the laser beam to the linac interaction region will proceed in parallel. First demonstration of Thomson scattered light from the linac beam and FALCON laser pulses represents the key milestone of the project.

\section{ACKNOWLEDGMENTS}

Work performed under the auspices of the U.S. Dept. of Energy by the Lawrence Livermore National Laboratory under Contract W-7405-Eng-48.

\section{REFERENCES}

1. R.W. Shoenlein, et al., 'Femtosecond $\mathrm{x}$-ray pulses at 0.4 angstrom generated by 90 degree Thomson scattering: A tool for probing the structural dynamics of materials,"Science274, 236 (1996).

2. W.P. Leemans et al., "Interaction of relativistic electrons with ultrashort laser pulses: Generation of femtosecond x-rays and microprobing of electron beams," IEEE Jour. of Quant. Elec. 33, 1925 (1997).

3. D.T. Palmer et al., "Emittance studies of the BNL/SLAC/UCLA 1.6 cell photocathode rf gun," IEEE Part. Accel. Conf. (1997).

4. H. Matsumoto et al., "Applications of hot isostatic pressing (HIP) for high gradient accelerator structure," Proc. PAC 911008 (1992).

5. D. Gooden and J. Rosenzweig, "Modeling of the $\mathrm{x}$-ray radiation dependence on power in high gradient radiofrequency accelerator structures," UCLA Part. Beam Phys. Lab. Internal Report (1999).

6. X.-J. Wang et al., "Intense electron emission due to picosecond laser produced plasmas in high gradient electric fields,"Journal of Applied Physics, 72(3), p. 888-894 (1992).

7. J. Rosenzweig and G. Travish, "Design Considerations for the UCLA PBPL Slit-based Phase Space Measurement Systems," Nucl. Instrum. Meth. A 341, p. 379 (1994).

8. T. Ditmire and M.D. Perry, "High intensity physics with a table-top 20 TW laser system", LLNL Internal Report, UCRL-ID-133293 (1999).

9. V.P. Yanovsky et al., "Multiterawatt Laser-Linac Facility," Conference on Lasers and Electron Optics OSA Technical Digest (OSA, Washington DC), 410 (1999).

10. T. Kotseroglou et al., "Picosecond timing of terawatt laser pulses with the SLAC 46 GeV electron beam," Nucl. Instrum. Meth. A 383, 309-317 (1996).

11. M.J.W. Rodwell, D.M. Bloom, K.J. Weingarten, "Subpicosecond laser timing stabilization," IEEE J. Quantum Electronics 25, 817 (1989).

12. J.F. Schmerge et al., "Photocathode if gun emittance measurements using variable length laser pulses," SPIE Photonics West, LASE 99, Proceedings of SPIE Vol. 3614

13. E. Esarey et al, "Nonlinear Thomson Scattering of Intense Laser Pulses from Beams and Plasmas,"Phys. Rev. E 48, 3003 (1993).

14. Yu-Jiuan Chen, personal communication.

15. G.P. Le Sage st al., "Transverse phase space mapping of relativistic electron beams using optical transition radiation," Phys. Rev. Spec. Topics - Accel. And Beams 2, 122802 (1999). 